Article

\title{
Bio-inspired synthesis of reduced graphene oxide wrapped Geobacter sulfurreducens as a hybrid electrocatalyst for efficient oxygen evolution reaction
}

Shafeer Kalathil, Krishna P. Katuri, Amira S. Alazmi, Srikanth Pedireddy, Nikolay Kornienko, Pedro M. F. J. Costa, and Pascal E. Saikaly

Chem. Mater., Just Accepted Manuscript • DOI: 10.1021/acs.chemmater.9b00394 • Publication Date (Web): 17 Apr 2019

Downloaded from http://pubs.acs.org on April 25, 2019

\section{Just Accepted}

"Just Accepted" manuscripts have been peer-reviewed and accepted for publication. They are posted online prior to technical editing, formatting for publication and author proofing. The American Chemical Society provides "Just Accepted" as a service to the research community to expedite the dissemination of scientific material as soon as possible after acceptance. "Just Accepted" manuscripts appear in full in PDF format accompanied by an HTML abstract. "Just Accepted" manuscripts have been fully peer reviewed, but should not be considered the official version of record. They are citable by the Digital Object Identifier (DOI®). "Just Accepted" is an optional service offered to authors. Therefore, the "Just Accepted" Web site may not include all articles that will be published in the journal. After a manuscript is technically edited and formatted, it will be removed from the "Just Accepted" Web site and published as an ASAP article. Note that technical editing may introduce minor changes to the manuscript text and/or graphics which could affect content, and all legal disclaimers and ethical guidelines that apply to the journal pertain. ACS cannot be held responsible for errors or consequences arising from the use of information contained in these "Just Accepted" manuscripts. 


\section{Bio-inspired synthesis of reduced graphene oxide wrapped Geobacter sulfurreducens as a hybrid electrocatalyst for efficient oxygen evolution reaction}

Shafeer Kalathil ${ }^{1}$, Krishna P. Katuri ${ }^{1}$, Amira S. Alazmi², Srikanth Pedireddy ${ }^{1}$, Nikolay Kornienko ${ }^{3}$, Pedro M. F. J. Costa², Pascal E. Saikaly ${ }^{*}$

${ }^{1}$ Division of Biological and Environmental Sciences \& Engineering, Water Desalination and Reuse Center, King Abdullah University of Science and Technology, Thuwal, Saudi Arabia $23955-6900$

${ }^{2}$ Division of Physical Sciences and Engineering, King Abdullah University of Science and Technology, Thuwal, Saudi Arabia 23955-6900

${ }^{3}$ Department of Chemistry, Université de Montréal, Roger-Gaudry Building, Montreal, Quebec, H3C 3J7, Canada

* To whom correspondence should be addressed. E-mail: pascal.saikaly@,kaust.edu.sa 


\begin{abstract}
Doping/decorating of graphene or reduced graphene oxide (rGO) with heteroatoms provides a promising route for the development of electrocatalysts useful in many technologies, including water splitting. However, current doping approaches are complicated, not eco-friendly and not costeffective. Herein, we report the synthesis of doped/decorated rGO for oxygen evolution reaction (OER) using a simple approach that is cost-effective, sustainable and easy to scale up. The OER catalyst was derived from the reduction of GO by an exo-electron transferring bacterium, Geobacter sulfurreducens. Various analytical tools indicate that OER active elements such as $\mathrm{Fe}, \mathrm{Cu}, \mathrm{N}, \mathrm{P}$, and S decorate the rGO flakes. The hybrid catalyst (i.e., Geobacter/rGO) produces a geometric current density of $10 \mathrm{~mA} \mathrm{~cm}^{-2}$ at an overpotential of $270 \mathrm{mV}$ vs. the reversible hydrogen electrode (RHE) with a Tafel slope of $43 \mathrm{mV} \mathrm{dec}{ }^{-1}$, and possesses high durability, evidenced through 10 hours of stability testing. Electrochemical analyses suggest the importance of Fe and its possible role as active site for OER. Overall, this work represents a simple approach towards the development of earthabundant, eco-friendly and highly active OER electrocatalyst for various applications such as solar fuel production, rechargeable metal-air batteries, and microbial electrosynthesis.
\end{abstract}




\section{Introduction}

The world is facing a rapidly growing demand for energy. Currently, energy production depends mainly on fossil fuels, ${ }^{1}$ which are being rapidly depleted and are a major cause of climate change. Hence, there is a need to explore alternative energy sources where the renewable sources stand out as the most attractive. ${ }^{1}$ Amongst these, electrons extracted from water via water oxidation can be used to make molecular hydrogen or high-value chemicals/fuels from carbon dioxide $\left(\mathrm{CO}_{2}\right)$ reduction. ${ }^{2}$

Water splitting is a key process in solar fuel production, ${ }^{3}$ rechargeable metal-air batteries, ${ }^{4}$ and microbial electrosynthesis. ${ }^{5-7}$ One of the main challenges in water splitting is that water oxidation needs a high energy input due to its kinetically sluggish multi-step reaction pathway. ${ }^{8}$ Hence, stable, highly active and low-cost water oxidation catalysts should be developed for the wide-scale application of this process for energy production. Currently, metal oxide-based compounds such as $\mathrm{IrO}_{2}$ and $\mathrm{RuO}_{2}$ show the highest water oxidation activities with low overpotential at $10 \mathrm{~mA} \mathrm{~cm}^{-2} .8$ However, these materials suffer from multiple disadvantages such as scarcity, high cost and poor stability. ${ }^{8}$ Due to their relatively high conductivity, mechanical stability, widespread availability and low toxicity, graphene flakes bear great promise for electrocatalytic purposes. ${ }^{8,9}$ Whilst the catalytic performance of exfoliated graphene is poor, it can be improved through functionalization with heteroelements to create highly active catalytic sites. ${ }^{9}$ Recently, the doping/decorating of graphene with nonmetals (e.g., S, N, P, F and O $)^{9-13}$ and metals ${ }^{8}$ (e.g., Fe and Ni) has proven to be a feasible strategy to render it an efficient electrocatalyst for oxygen evolution reaction (OER), hydrogen evolution reaction (HER) and oxygen reduction reaction (ORR). In the case of graphene doping with nitrogen (Ngraphene), ${ }^{9}$ the substitutional element influences the spin density and charge distribution in the adjacent carbon atoms. Overall, this creates an "activation region" on the graphene surface which can 
subsequently participate in various catalytic reactions. ${ }^{14,15}$ There are several ways to produce $\mathrm{N}$ graphene materials through direct and post-synthesis methods ${ }^{9}$ (summarized in Table S1).

Sulfur $(\mathrm{S})$ and phosphorous $(\mathrm{P})$ are other elements that can be introduced into graphene. ${ }^{10,13}$ As their size and electronegativity are largely different from carbon, these elements can induce structural distortions and locally change the electronic structure of graphene. In the presence of a precursor containing S, the chemical vapor deposition (CVD) approach can be used to produce S-doped graphene (S-graphene). ${ }^{9}$ P-doped graphene (P-graphene) can be prepared by treating graphene oxide (GO) with phytic acid at high temperature. ${ }^{10}$ The doping of graphene with several elements at the same time, as for example, doping with $\mathrm{N}$ and $\mathrm{S}$ or $\mathrm{N}, \mathrm{S}$ and $\mathrm{P}$ can further enhance the catalytic activity due to synergetic effects. ${ }^{12,16,17}$ While not exhaustive, the above-mentioned strategies represent the most common approaches to produce doped graphene. Generally, these methods suffer from multiple drawbacks such as high cost, use of complicated synthesis procedures (high temperature and use of gases) and toxic precursors, and the release of hazardous waste-products (Table S1).

Green synthesis of reduced $\mathrm{GO}(\mathrm{rGO})$ from $\mathrm{GO}$ has been demonstrated using pure cultures of extracellular electron transfer (EET)-capable bacteria, such as Shewanella oneidensis MR-1, ${ }^{18,} 19$ Bacillus subtilis $168,{ }^{20}$ Gluconobact roseus, ${ }^{21}$ and Desulfovibrio desulfuricans. ${ }^{22}$ EET-capable bacteria have the ability of coupling the oxidation of substrates (electron donor) in their cytoplasm with the reduction of insoluble extracellular electron acceptors for respiration. The molecular mechanism of electron transfer to GO by EET-capable bacteria was only reported by few studies, where electron transfer to GO was mediated either directly by outer membrane cytochromes as in Shewanella oneidensis MR-1 $1^{18,19}$ or indirectly by redox mediator (e.g., vitamin $\mathrm{K}_{3}$ ) as in Bacillus subtilis $168 .^{20}$ The reduction of GO to $\mathrm{rGO}$ was also been reported using mixed-culture inoculum such as anaerobic 
sludge, ${ }^{23}$ river water, ${ }^{24}$ water channel sediment and paddy soil, ${ }^{24}$ with the main objective to demonstrate the applicability of GO for the selective enrichment of EET-capable bacteria from the environment.

As living organisms, bacteria are mainly composed of carbon (50\% on dry weight basis) with abundant heteroatoms (e.g., N, P, and S) and trace elements. ${ }^{25}$ Therefore, bacteria can provide abundant heteroatoms to dope carbon materials. The production of carbon dot-heteroatom $(\mathrm{N}, \mathrm{S}$, and P) doped rGO for ORR was recently been demonstrated in a two-step process using the EET-capable bacterium S. oneidensis MR-1, where GO is reduced to $\mathrm{rGO}$ by $S$. oneidensis MR-1 in the first step followed by hydrothermal treatment at $180{ }^{\circ} \mathrm{C}$ in the second step. ${ }^{26}$

Inspired by these studies, here we developed an eco-friendly and highly efficient OER catalyst by utilizing for the first time the EET capability of the non-pathogenic bacterium, Geobacter sulfurreducens, to convert GO to rGO while simultaneously doping/decorating graphene with nonmetallic $(\mathrm{N}, \mathrm{S}$ and $\mathrm{P})$ and transition metal species $(\mathrm{Fe}$ and $\mathrm{Cu})$ derived from $G$. sulfurreducens in a single step. The rationale for selecting G. sulfurreducens is because it is commonly detected as a dominant member of anodic community in microbial electrochemical systems due to its outstanding performance in generating electricity (using conductive electrodes as electron acceptor) through substrate oxidation. ${ }^{27,} 28$ The unique ability of G. sulfurreducens to make contact with insoluble electron acceptors is because it possesses a vast network of multi-haem containing outer-membrane ctype cytochromes (OM c-Cyts) and nano-filaments (known as microbial protein nanowires). ${ }^{27,}{ }^{29} \mathrm{It}$ employs OM c-Cyts to transfer metabolically generated electrons from its periplasm to the cell exterior through a well-orchestrated EET process. ${ }^{30}$ Iron is a key component of haem group in c-Cyts. The content of iron in G. sulfurreducens cell is three-fold higher compared to Escherichia coli, which is the best bacteria reported in terms of iron assimilation. ${ }^{31}$ This is mainly due to the fact that $G$. 
sulfurreducens possesses the highest number of genes coding for c-Cyts (111 in the whole genome) ${ }^{32}$ Thus, this bacterium assimilates more iron into their cell for synthesizing c-Cyts required for EET mechanism. Moreover, in a recent study it was shown that iron and not nickel was performing watersplitting in a catalyst made of layers of nickel and iron. ${ }^{33}$ In addition to c-Cyts, G. sulfurreducens possesses multicopper containing proteins ${ }^{34}$ and $\mathrm{Fe}-\mathrm{S}$ protein clusters ${ }^{30}$. Taken together, these characteristics make G. sulfurreducens ideally suited for generating metal and non-metal doped/decorated rGO for electrocatalysis such as OER.

The synthesis protocol of doped/decorated rGO using G. sulfurreducens overcomes most drawbacks of other existing methods aimed at the production of doped/decorated graphene for electrocatalysis (Table S1). The hybrid catalyst (i.e., Geobacter/rGO) demonstrated high electrocatalytic activity towards OER in alkaline electrolyte, producing a geometric current density of $10 \mathrm{~mA} \mathrm{~cm}{ }^{-2}$ at an overpotential of $270 \mathrm{mV}$ vs. the reversible hydrogen electrode (RHE).

\section{Experimental Procedures}

\section{Synthesis of graphene oxide (GO) and hydrothermally reduced graphene oxide (HrGO):}

Graphite powder (Alfa Aesar, $<50 \mu \mathrm{m}$ ) was first oxidized using the so-called Improved-Hummers' method. ${ }^{35}$ Typically, $3 \mathrm{~g}$ of graphite powder (Alfa Aesar) was added to a mixture of $360 \mathrm{~mL} \mathrm{H}_{2} \mathrm{SO}_{4}$ (Sigma Aldrich, 99\%) and $40 \mathrm{~mL} \mathrm{H}_{3} \mathrm{PO}_{4}$ (Sigma Aldrich, $85 \mathrm{wt} \%$ ). This was followed by the slow addition of $18 \mathrm{~g}$ of $\mathrm{KMnO}_{4}$ (Acros, 99\%), taking care that the reaction temperature was maintained at $<20{ }^{\circ} \mathrm{C}$. Then, the resulting suspension was heated, in an oil bath, to $50{ }^{\circ} \mathrm{C}$ and stirred for $12 \mathrm{~h}$. The coluor of the mixture turned from black to mud-brown. The reaction was allowed to cool to room temperature and treated with $400 \mathrm{~mL}$ of cold deionized water plus $3 \mathrm{~mL}$ of $\mathrm{H}_{2} \mathrm{O}_{2}$ (Sigma Aldrich, $30 \%$ ). Then, the product was diluted with deionized water to a total volume of 2 L. Finally, the 
suspension was repeatedly washed with water and centrifuged (Hettich U320, $9000 \mathrm{rpm}, 10 \mathrm{~min}$ ) until the $\mathrm{pH}$ was nearly neutral. At this point, the suspension was vacuum dried in the centrifuge tubes (60 $\left.{ }^{\circ} \mathrm{C}, 12 \mathrm{~h}\right)$ and the resulting powder collected. The graphene oxide $(\mathrm{GO})$ solution $\left(5 \mathrm{mg} \mathrm{ml}^{-1}\right)$ was prepared by dispersing the GO powder in deionized water and then the mixture was stirred for 24 hours. Hydrothermally reduced graphene oxide (HrGO) was prepared according to a previous report. ${ }^{36}$ Reduction of GO to reduced graphene oxide (Geobacter/rGO) by G. sulfurreducens: G. sulfurreducens PCA (ATCC 51573) was used as the bacterium for the synthesis of Geobacter/rGO. G. sulfurreducens was cultured in an anaerobic serum bottle using acetate $(20 \mathrm{mM})$ as electron donor and fumarate $(50 \mathrm{mM})$ as the electron acceptor in defined media. ${ }^{37}$ The entire culturing was conducted in an anaerobic glove box and the bottle was then kept in a shaking incubator $\left(30^{\circ} \mathrm{C}\right)$ for five days. Although strict anaerobic conditions are not required as G. sulfurreducens can survive in oxic environments, ${ }^{38}$ we maintained anaerobic conditions for the experiment to avoid electron consumption by oxygen which may interfere in the GO reduction.

As grown G. sulfurreducens suspension (100 ml) having an optical density (OD) of 0.8 was centrifuged and the resulting pellet appeared red in colour due to the presence of multi-heme containing outer-membrane c-type cytochromes (OM c-Cyts). ${ }^{39}$ The bacterial pellet was added to an anaerobic serum bottle that contains G. sulfurreducens growth media (100 ml, except fumarate) with $20 \mathrm{mM}$ acetate (electron donor) and GO $\left(0.4 \mathrm{mg} \mathrm{mL}^{-1}\right)$ as the sole source of electron acceptor. The bottle was kept in a temperature-controlled room $\left(30{ }^{\circ} \mathrm{C}\right)$ for 2 days without shaking. Several batch experiments were conducted to reproduce the data. Three serum bottles were kept under similar conditions but without inoculation of G. sulfurreducens (denoted as abiotic GO). To understand the role of OM c-Cyts in the GO reduction, G. sulfurreducens was grown with a medium containing 30 
$\mu \mathrm{M}$ of the iron chelator, 2,2'-bipyridine to suppress the production of OM c-Cyts. ${ }^{40}$ The cells grown with bipyridine were then employed for the GO reduction. Heat-killed bacteria were prepared by autoclaving bacterial solution. The autoclaved bacterial cells were used as the inoculum to reduce GO to understand the metabolic activity of bacteria in the GO reduction.

Geobacter/rGO and abiotic GO processing: After two days of G. sulfurreducens incubation with GO, a dark colored hydrogel type Geobacter/rGO was precipitated at the bottom of the bottle. The solution underwent centrifugation ( $8000 \mathrm{rpm}, 6$ minutes) to collect the Geobacter/rGO material. The material was washed six times with MilliQ water by gentle vortexing to remove any media contribution on the Geobacter/rGO. After the washing process, Geobacter/rGO was kept in an oven $\left(45^{\circ} \mathrm{C}\right)$ overnight for drying the sample. The dried sample was used for all experiments. There was no GO reduction in the absence of G. sulfurreducens under similar conditions even after 2 days of the incubation (abiotic GO). The abiotic GO was collected by centrifugation (14000 rpm, 10 minutes) and the collected sample was washed with MilliQ water (6 times) to remove any possible media contribution in the sample. The collected abiotic GO sample was dried at $45^{\circ} \mathrm{C}$ in an oven overnight for drying. The dried abiotic GO sample was used for all the experiments.

Materials Characterization: The elements are quantified using an ICP-OES Varian 720-ES Spectrometer equipped with a dual detector assembly that covered a wavelength from 165 to $782 \mathrm{~nm}$. Raman spectra were collected using a WITec Alpha300RA spectrometer at an excitation wavelength of $488 \mathrm{~nm}$. The survey and high-resolution X-ray photoelectron spectroscopy (XPS) spectra were obtained at fixed analyzer pass energies of $160 \mathrm{eV}$ and $20 \mathrm{eV}$, respectively (Model: ESCA 3400). Transmission electron microscopy (TEM) images were acquired in bright-field mode with a Phillips Tecnai F20 electron microscope with $200 \mathrm{kV}$ accelerating voltage. Samples were prepared by 
sonicating in ethanol and drop-casting on an ultrathin carbon grid, followed by drying under ambient conditions. Contact angle was measured using a FTA1000 B Class goniometer (First Ten Ångstroms), paired with FTA32 software. Scanning electron microscopy (SEM) images were acquired using a Zeiss Merlin microscope under an acceleration voltage of $5 \mathrm{keV}$. The SEM-EDS measurements were obtained by using an Oxford EDS X-Max SDD detector attached to the Zeiss Merlin scanning electron microscope, and the AzTecEnergy EDS analysis software. Fourier-Transform Infrared (FTIR) spectra were obtained by a Thermo Scientific ${ }^{\mathrm{TM}}$ Nicolet $^{\mathrm{TM}}$ iS50 FTIR Spectrometer at ATR mode.

Electrochemical tests: The activity of the Geobacter/rGO towards the OER was tested using a rotating disc electrode (RDE). The working electrode was prepared by the following procedure: first, the Geobacter/rGO material ( $2 \mathrm{mg}$ ) was dispersed in $500 \mu \mathrm{l}$ of ethanol, $500 \mu \mathrm{l}$ of water and $15 \mu 1$ of Nafion (as binder). The dispersed solution was sonicated for $30 \mathrm{~min} .2 \mu 1$ of the obtained slurry was drop-coated onto a $3 \mathrm{~mm}$ glassy carbon disc electrode (GCE; loading concentration $\sim 0.05 \mathrm{mg} \mathrm{cm}^{-2}$ ) and dried under a lamp for $1 \mathrm{~h}$. The same procedure and loading concentration were used to make working electrodes with hydrothermally reduced GO (HrGO), abiotic GO and pure G. sulfurreducens dried cells. The electrochemical measurement was carried out using a BioLogic VMP3 electrochemical working station in $1 \mathrm{M} \mathrm{KOH}$ (Sigma Aldrich, semiconductor grade, pellets, 99.99\% trace metals basis) at room temperature using a three-electrodes system, in which $\mathrm{Pt}$ coil and Mercury/Mercury oxide $(\mathrm{Hg} / \mathrm{HgO})$ were used as counter and reference electrodes, respectively. Linear sweep voltammetry (LSV) experiments were performed at a scan rate of $5 \mathrm{mV} \mathrm{s}^{-1}$ while maintaining a constant rotational speed of $1600 \mathrm{rpm}$. Stability test for the Geobacter/rGO OER catalyst was performed by conducting chronoamperometry measurement (CA) at an overpotential of $270 \mathrm{mV}$ vs. reversible hydrogen electrode (RHE) under a constant rotating speed of $1600 \mathrm{rpm}$. Cyclic voltammetry 
(CV) analysis was conducted at a scan rate of $5 \mathrm{mV} \mathrm{s}^{-1}$ without rotation. Electrochemical impedance spectroscopy (EIS) measurements were performed for the Geobacter/rGO in $1 \mathrm{M} \mathrm{KOH}$ from 1000 $\mathrm{kHz}$ to $100 \mathrm{mHz}$. All the measured potentials vs. the $\mathrm{Hg} / \mathrm{HgO}$ were converted to RHE by the Nernst equation $\left(\mathrm{E}_{\mathrm{RHE}}=\mathrm{E}_{\mathrm{Hg} / \mathrm{HgO}}+0.0591 \mathrm{pH}+0.140\right)$. All the experiments were done at least in triplicates to reproduce the data.

\section{Results and discussion}

An active culture of $G$. sulfurreducens, with acetate $(20 \mathrm{mM})$ as the electron donor and GO $\left(0.4 \mathrm{~g} \mathrm{~L}^{-1}\right)$ as the electron acceptor, was used (Fig. 1a). The culture was incubated under anaerobic conditions for 2 days at $30^{\circ} \mathrm{C}$ to permit the complete reduction of the brownish GO and formation of a black hydrogel (lower inset in Fig. 1c). The formation of a hydrogel was previously reported for a pure culture of Geobacter sp. strain R4 when cultivated with acetate and GO $\left(0.67 \mathrm{~g} \mathrm{~L}^{-1}\right)$ at $28{ }^{\circ} \mathrm{C}$; however it required a longer incubation period $(30 \mathrm{~d})$ to form a hydrogel. In contrast, hydrogel formation was not observed with other EET-capable bacteria such as Shewanella oneidensis MR-1, Bacillus subtilis 168, Gluconobact roseus, or Desulfovibrio desulfuricans when incubated with GO (0.2-0.5 $\left.\mathrm{g} \mathrm{L}^{-1}\right)$ under anaerobic conditions for 1 day $^{20,22}$ or 3 days. ${ }^{18}$

Control experiments were done under similar conditions but in the absence of $G$. sulfurreducens (herewith termed abiotic GO; see details in Supporting Information). These control experiments confirmed that there was no reduction of GO without G. sulfurreducens in the solution. We employed scanning electron microscopy (SEM) to investigate the morphology of the as prepared Geobacter/rGO hydrogel. SEM images demonstrated that the Geobacter cells were embedded in the crosslinked ultrathin sheets of rGO matrix. Moreover, 
Geobacter cells were wrapped with rGO sheets indicating strong interaction between Geobacter and rGO sheets (Fig. 1c). The dimensions of the cells in the Geobacter/rGO hydrogel are in accordance with the size of Geobacter cells (Fig. 1b).

To understand the role of OM c-Cyts in the reduction of GO, we suppressed the production of OM c-Cyts by adding bipyridine (an iron-chelator) in the G. sulfurreducens growth media. Bipyridine is known to shut down the production of Cyts by chelating with iron. ${ }^{40}$ Inductively coupled plasma optical emission spectrometry (ICP-OES) analysis showed significantly lower Fe in the supressed cells compared to unsuppressed cells (Fig. S1). The OM c-Cyts suppressed cells did not reduce GO (Fig. S2) suggesting that OM c-Cyts are essential for GO reduction by G. sulfurreducens. Also, heat-killed bacteria were not able to reduce GO. This observation indicates that the G. sulfurreducens physiological activity was responsible for the reduction of GO as the electrons generated metabolically, via acetate oxidation, were transferred extracellularly to GO (which acts as the terminal electron acceptor) by OM c-Cyts, reducing it to rGO.

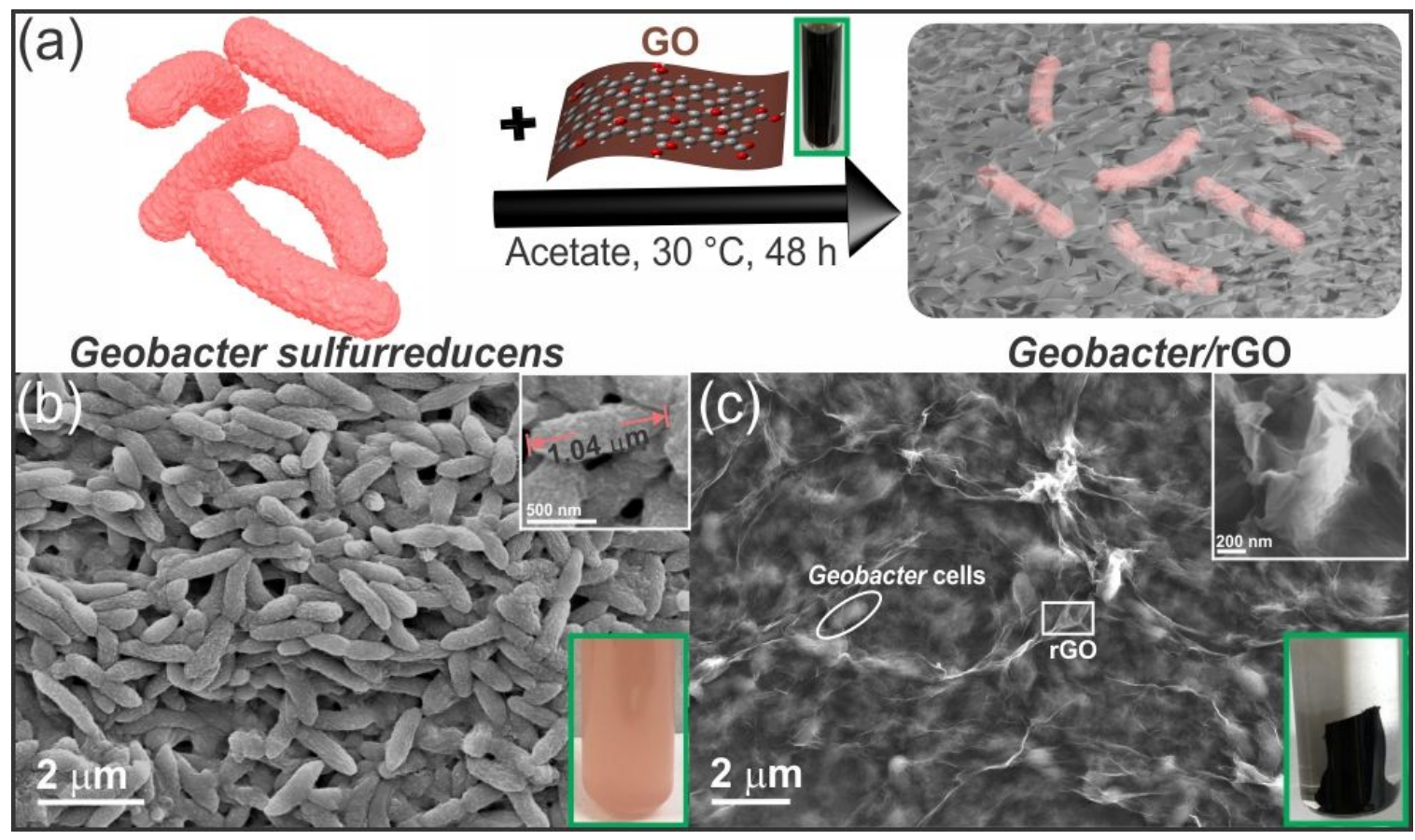

Figure 1. Schematic illustration and morphological characterization. (a) Schematic illustration for the reduction of graphene oxide (GO) by G. sulfurreducens (conditions: $30{ }^{\circ} \mathrm{C}$, anaerobic, $\mathrm{pH} 7$ ). 
(b) Low and high magnification (upper inset) Field-Emission Scanning Electron Microscope (FESEM) images of dried G. sulfurreducens cells and a digital photograph of the suspended $G$. sulfurreducens cells (lower inset). The red color is due to the iron-rich content in G. sulfurreducens. (c) Low and high magnification (upper inset) FE-SEM images of Geobacter/rGO and a digital photograph of the Geobacter/rGO hydrogel (lower inset) at the bottom of the anaerobic serum vial after $48 \mathrm{~h}$ of incubation.

Additional evidence for the successful reduction was acquired through spectroscopic observations (Fig. 2). We thoroughly investigated the Geobacter/rGO sample in comparison to GO using X-ray photoelectron spectroscopy (XPS) analysis. The high resolution C1S spectra of the Geobacter/rGO and abiotic $\mathrm{GO}$ (Fig. 2a) were significantly different, with the $\mathrm{C}=\mathrm{C}$ peak being clearly dominant in the former. The $\mathrm{C} / \mathrm{O}$ atomic ratio in Geobacter/rGO and abiotic $\mathrm{GO}$ were calculated to be 5.5 and 2.78, respectively (Figure S3 and S4). The increased C/O ratio in Geobacter/rGO confirmed the successful reduction of GO by G. sulfurreducens. After the GO reduction, the Geobacter/rGO showed presence of nitrogen (5\%) (Fig. 2c and Fig. S3) which may be derived from G. sulfurreducens (Fig. S5). Further confirmation was provided by Raman analysis. In the Raman spectra of abiotic GO and Geobacter/rGO (Fig. 2d), the D band is considerably narrower in Geobacter/rGO and the set of peaks at higher wavenumber $\left(2500-3500 \mathrm{~cm}^{-1}\right)$ were more resolved. The $\mathrm{I}_{\mathrm{D}} / \mathrm{I}_{\mathrm{G}}$ ratio of Geobacter $/ \mathrm{rGO}(1.18)$ was higher than that of abiotic $\mathrm{GO}(0.93)$. The increased $\mathrm{I}_{\mathrm{D}} / \mathrm{I}_{\mathrm{G}}$ ratio implies that there are less in-plane $\mathrm{sp}^{2}$ bonds and more out of plane $\mathrm{sp}^{3}$ bonds in the carbon atoms within the material, which may originate from the inclusion of the dopants. ${ }^{41}$ On the other hand, the increased peak intensity in the high wavenumber region, corresponding to the $2 \mathrm{D}, \mathrm{D}+\mathrm{G}$ and $2 \mathrm{G}$ bands may indicate a higher degree of disruption of the graphitic $\mathrm{AB}$ stacking order in Geobacter/rGO. ${ }^{42}$ Also, the $\mathrm{G}$ band of Geobacter/rGO blue-shifted possibly due to defects or N-doping by G. sulfurreducens (Fig. 2d) (Fig. 2d). ${ }^{43}$ Taken together, these results confirm the modulation of GO by G. sulfurreducens. 

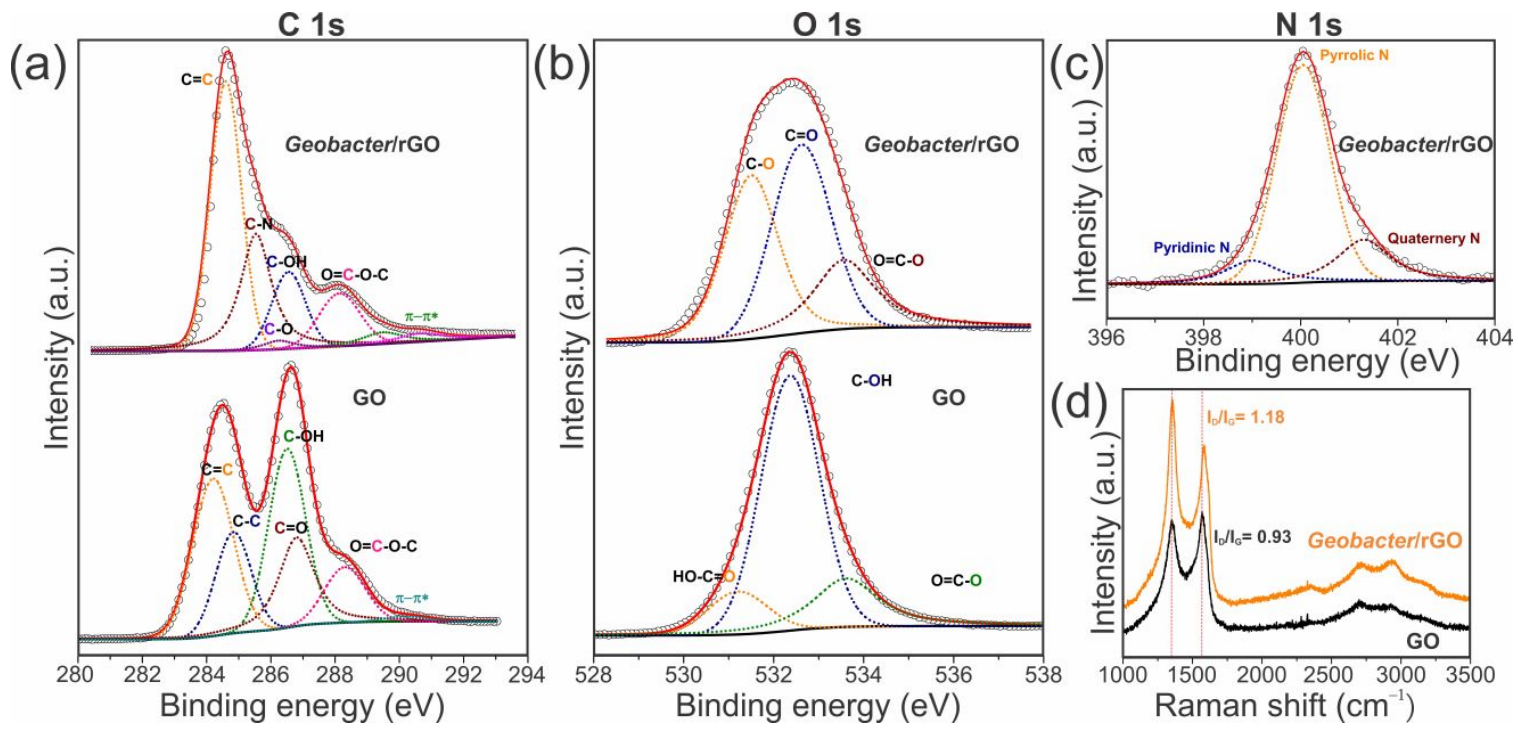

Figure 2. Comparative X-ray photoelectron spectroscopy (XPS) and Raman spectra of GO vs. Geobacter/rGO. Fitted XPS spectra of GO vs. Geobacter/rGO for (a) C 1s region, (b) O 1s region, and (c) N 1s region. (d) Raman spectra of GO and Geobacter/rGO.

Transmission electron microscopy (TEM) was utilized to examine the chemical and structural nature of Geobacter/rGO (Fig. 3). The low magnification TEM image of Geobacter/rGO clearly demonstrated the formation of interlinked network of rGO with embedded Geobacter cells (Fig. 3a). Presumably, the majority of the iron species are in the form of small clusters or in an amorphous phase. However, iron oxide (FEO) nanoparticles were occasionally seen (Fig. 3b). The lattice spacing, calculated from the Fast Fourier Transform (FFT) image, of 1.3 and $1.1 \AA$ is close to FeO (111) and (200) planes (lower inset of Fig. 3b). The inverse of the FFT is overlaid atop the original image to highlight the FeO particle. The Geobacter/rGO consisted of primarily single to few-layer morphology (Fig. 3c-d). The interlayer spacing between the individual sheets shrunk from $4.5 \AA$ for the GO precursor (Fig. S6b) to $3.6 \AA$ (Fig. 3e), pointing to the reduction of GO to Geobacter/rGO. The FFT reconstruction of the Geobacter/rGO illustrates the honeycomb carbon lattice, as expected for rGO (Fig. 3f). 
Multiple points of evidence indicated that the Geobacter/rGO was doped/decorated with elements beneficial for efficient OER. The ICP-OES analysis of G. sulfurreducens cells revealed that this bacterium was abundant with $\mathrm{S}, \mathrm{P}, \mathrm{Fe}$ and $\mathrm{Cu}$ (Table S2). The ICP-OES analysis of the Geobacter/rGO also showed the presence of these elements which may be derived from G. sulfurreducens (Table S2). XPS analysis showed $11.9 \% \mathrm{~N}$ (wt. \%) in the pure G. sulfurreducens, $5 \% \mathrm{~N}$ in the Geobacter/rGO and no $\mathrm{N}$ in pure GO (Fig. S3, S4 and S5). It should be noted that $\mathrm{S}, \mathrm{P}, \mathrm{Fe}, \mathrm{Cu}$ and $\mathrm{N}$ are excellent candidates for the OER. ${ }^{17,44-46}$

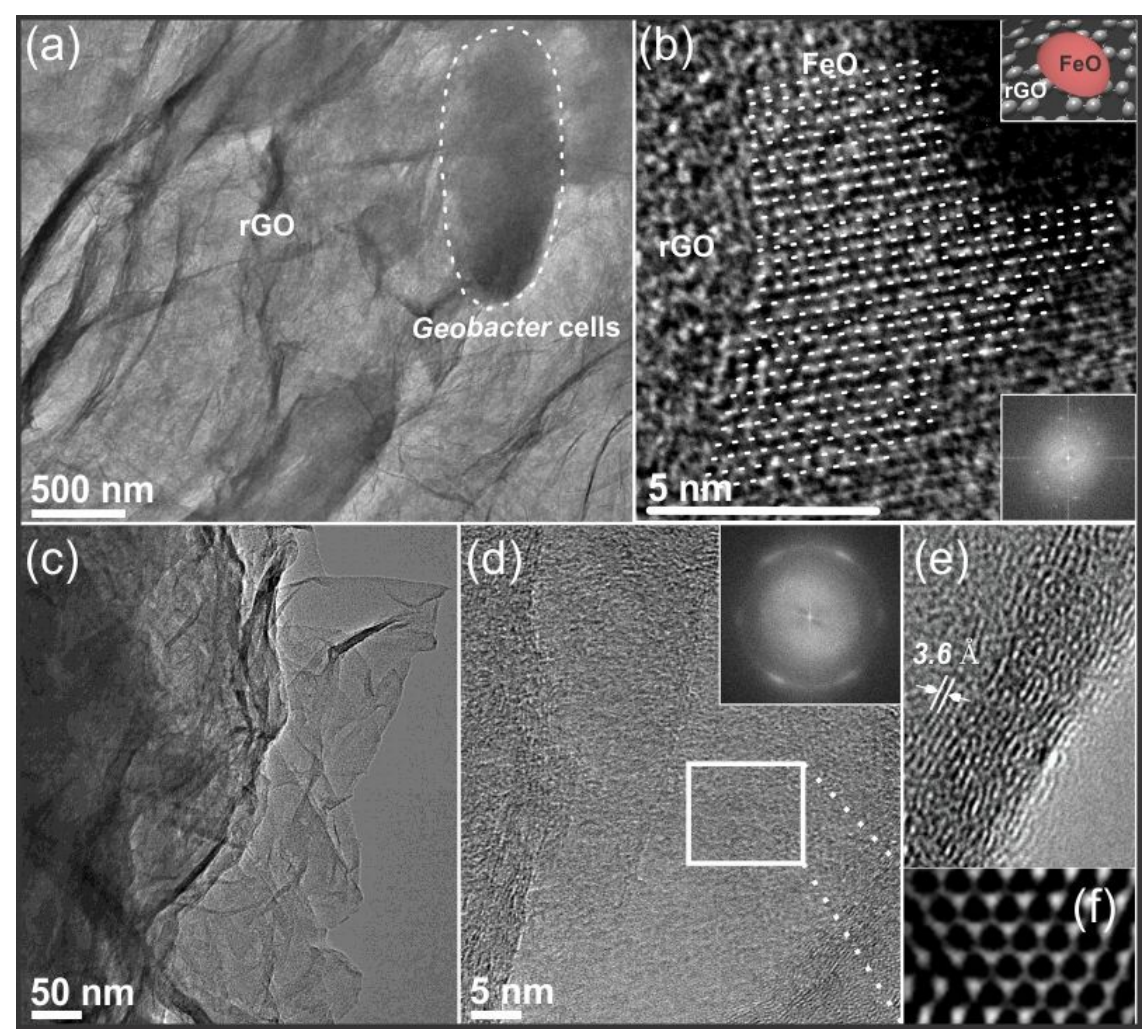

Figure 3. Structural characterizations of Geobacter/rGO using transmission electron microscopy (TEM). (a) TEM image of G. sulfurreducens cells embedded in rGO. (b) High-resolution TEM image and Fast Fourier Transform (FFT) pattern of rGO-FeO demonstrate the crystalline FeO. (c, d) Bright field TEM reveals single- to few-layer morphology of the Geobacter/rGO. The FFT inset of the single layer Geobacter/rGO illustrates the hexagonal arrangement of the carbon lattice. (e) A magnified image of several stacked Geobacter/rGO sheets reveals an interlayer spacing of $\sim 3.6 \AA$, 
pointing to a near-complete reduction of the GO precursor. (f) An inverse FFT of single Geobacter/rGO sheet showing the honeycomb carbon lattice.

After thoroughly characterizing the Geobacter/rGO, the electrocatalytic OER properties of Geobacter/rGO were evaluated using linear sweep voltammetry (LSV) (Fig. 4). To validate and compare its OER activity, control experiments were performed utilizing hydrothermally reduced graphene oxide $(\mathrm{HrGO})^{36}$ and abiotic GO. Working in $1 \mathrm{M} \mathrm{KOH}$ electrolyte, the Geobacter/rGO exhibited some OER activity while the other electrodes (built from $\mathrm{HrGO}$ and abiotic GO) showed minimal OER currents (Fig. 4a). Chronoamperometry (CA) experiments illustrated that the OER current generated by the Geobacter/rGO gradually increased, stabilising after $\sim 20 \mathrm{~h}$ (Fig. 4b). By contrast, there was no current produced with the $\mathrm{HrGO}$ and abiotic GO samples using similar mass loadings of $0.05 \mathrm{mg} \mathrm{cm}^{-2}$ (Fig. 4b). The Geobacter/rGO reached the highest OER performance with an overpotential of $270 \mathrm{mV}$ (vs. RHE) to produce a geometric current density of $10 \mathrm{~mA} \mathrm{~cm}^{-2}$ (Fig. 4c). The Tafel slope of $43 \mathrm{mV} \mathrm{dec}{ }^{-1}$ for Geobacter/rGO (Fig. 4d) is amongst the best for alkaline OER catalysts. ${ }^{47}$ The stark differences in Tafel slopes between the abiotic GO and Geobacter/rGO point to significant differences in per-site catalytic activity. There was no change in the OER activity of the Geobacter/rGO even after changing the used electrolyte for a fresh one (Fig. S7). The electrocatalytic inertness of $\mathrm{HrGO}$ and abiotic $\mathrm{GO}$ (even after the CA experiment) added to the absence of a significant change in the OER activity of the Geobacter/rGO after changing the electrolyte, rules out any possible role of impurities or platinum dissolution from the counter electrode. Also, impurities were not detected in the $\mathrm{KOH}$ (semiconductor grade) supporting electrolyte (Table S2) avoiding any possible electrolyte contamination during electrochemical experiments. Moreover, we did not observe any heteroatoms in the electrolyte after the CA experiment which confirms the robustness of the 
doped/decorated elements originated from the Geobacter/rGO (Table S2). The OER activity measured for the Geobacter/rGO is therefore solely attributed to this material. The low overpotential of $270 \mathrm{mV}$ demonstrates the OER activity of Geobacter/rGO is much higher than that of recently reported electrocatalysts and benchmark metal oxides such as $\mathrm{IrO}_{2}$ and $\mathrm{RuO}_{2}$ catalyst (Table $\mathrm{S} 3$ ). In addition, Geobacter/rGO catalyst was stable even after 10 hours of CA experiment (Fig. 4e). Control experiments performed with pure G. sulfurreducens cells showed negligible OER activity, even after an equivalent CA experiment (Fig. S8). As previously mentioned, multiple sources of evidence (Table S2 and Fig. 2c) demonstrate that the Geobacter/rGO was doped/decorated with species promoting the high OER activity of Geobacter/rGO electrocatalyst. In the Geobacter/rGO OER catalyst, $G$. sulfurreducens provided necessary OER elements to the rGO support which is distinct from HrGO and other rGO produced by various methods. To investigate the origin of this high activity in Geobacter/rGO electrocatalyst, we washed Geobacter/rGO sample with $1 \mathrm{M} \mathrm{HCl}$ solution to remove cell debris, organic matter and transition metals (such as Fe) as corresponding chlorides. Interestingly, acid washing diminished the catalytic activity of Geobacter/rGO electrocatalyst (Fig. 4c-d) indicating that Geobacter cells are crucial for attaining such a high catalytic performance. 
(a)

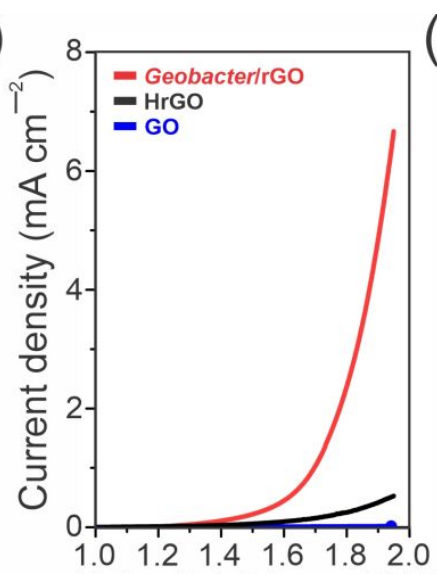

(d)

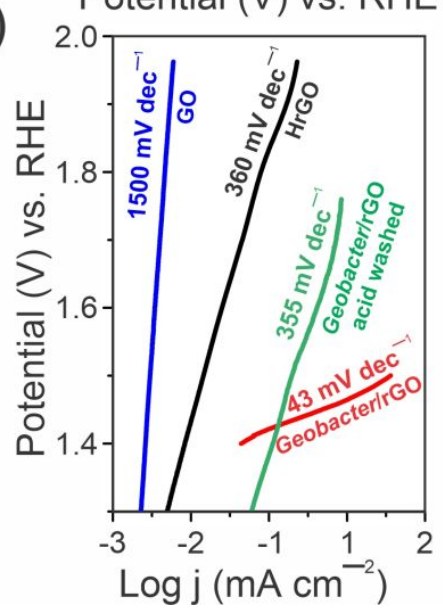

(b)

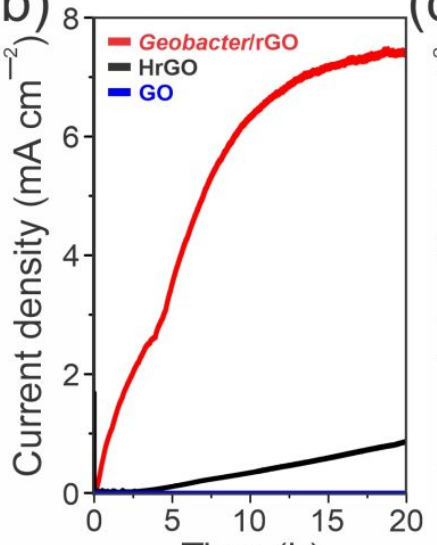

(e)

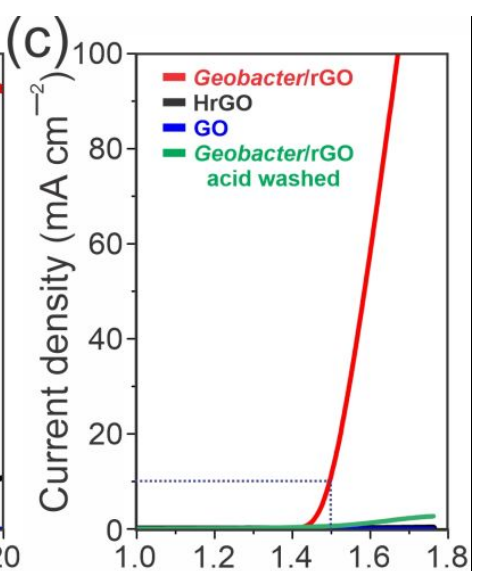

Figure 4. Electrochemical oxygen evolution reaction (OER) performance of GO, Geobacter/GO and HrGO deposited glassy carbon electrodes in $1 \mathrm{M} \mathrm{KOH} \mathrm{(pH} \mathrm{14)} \mathrm{solution.} \mathrm{(a)} \mathrm{Linear} \mathrm{sweep}$ voltammetry (LSV) plots normalized to geometric area of electrodes before chronoamperometry experiment at a scan rate of $5 \mathrm{mV} \mathrm{s}^{-1}$, (b) Chronoamperometry plots at an applied potential of $1.5 \mathrm{~V}$ vs RHE, (c) LSV plots after chronoamperometry experiment at a scan rate of $5 \mathrm{mV} \mathrm{s}^{-1}$, (d) Tafel plots of the used catalysts and (e) Stability test for Geobacter/rGO OER catalyst at an overpotential of 270 $\mathrm{mV}$ in $1 \mathrm{M} \mathrm{KOH}$. The rotating speed for all experiments was kept as $1600 \mathrm{rpm}$.

We performed several experiments to decipher the enhanced OER activity of Geobacter/rGO catalyst after the CA. SEM image of Geobacter/rGO catalyst after the CA showed the formation of several nanoparticles on the surface of the catalyst (Fig. 5a). A line-profile analysis using energydispersive spectroscopy (EDS) confirmed that these particles are made of FeO (Fig. 5b). The direction of the EDS line scan is denoted by a white arrow across the nanoparticle. The profile analysis indicates that $\mathrm{Fe}$ and oxygen atoms are distributed uniformly throughout the particle. Moreover, the intensity of 
the Fe component was higher compared to oxygen, and also oxygen concentration at the rims of the nanoparticle was slightly higher, suggesting surface oxidation is responsible for the formation of FeO.

ICP-OES analysis further confirmed that the hetero elements are intact with the catalyst even after the CA (Table S2). Also, the contact angle measurement and Fourier-transform infrared (FTIR) analysis showed that the Geobacter/rGO catalyst became more hydrophilic after the CA (Fig. 5c and 5d).

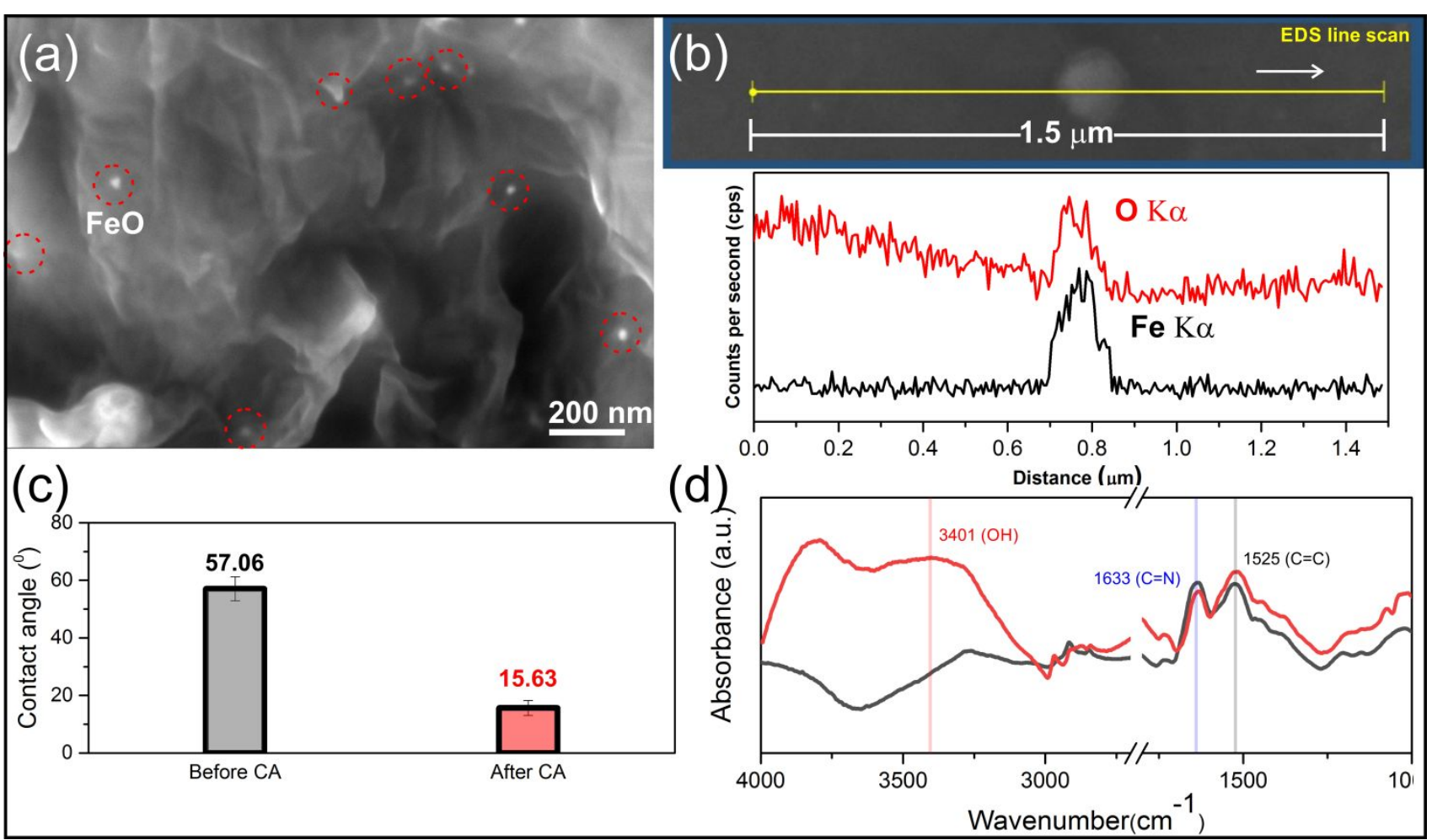

Figure 5. Scanning electron microscopy (SEM), Energy-dispersive spectroscopy (EDS), contact angle and Fourier-transform infrared (FTIR) characterization to evaluate changes in Geobacter/rGO catalyst after chronoamperometry (CA) measurement. (a) High magnification SEM image shows the presence of nanoparticles on the surface of Geobacter/rGO catalyst after the CA (b) EDS line profile analysis confirms that these nanoparticles are made of iron oxide (c) Contact angle and (d) FTIR spectroscopy measurement of Geobacter/rGO electrode before (black) and after (red) the CA. 
Complementary electrochemical impedance spectroscopy (EIS) analysis was conducted on the Geobacter/rGO electrode before and after the CA. The impedance spectra (Fig. S9) were fitted using a standard Randles circuit with EC-Lab V11.12 (Bio-Logic). Based on the Randles circuit analysis, the charge transfer resistances $\left(\mathrm{R}_{\mathrm{ct}}\right)$ were 10.3 (before $\mathrm{CA}$ ) and $0.76 \Omega$ (after CA) for the tested Geobacter/rGO sample. This result demonstrates that the conductivity of Geobacter/rGO was significantly enhanced after the CA experiment. Furthermore, cyclic voltammetry (CV) plot of Geobacter/rGO, done after the CA, showed a reversible oxidation peak (Fig. S10). This redox peak likely corresponds to the oxidation of Fe (decorated on the Geobacter/rGO, Fig. 5a and 5b) into its catalytically active $\mathrm{Fe}(\mathrm{III})$ and $\mathrm{Fe}(\mathrm{IV})$ states because this peak occurs just prior of OER catalysis and corresponds to previous reports. ${ }^{48}$ Note that the pair of redox peaks were absent in the corresponding CV before the CA (Fig. S10). The Geobacter/rGO exhibited a double-layer capacitance $\left(\mathrm{C}_{\mathrm{dl}}\right)$ of 14.29 and $2.14 \mathrm{mF} \mathrm{cm}^{-2}$ after and before $\mathrm{CA}$, respectively calculated from the $\mathrm{CVs}$ measured at non-Faradic region (Inset, Fig. S10). The enhanced $\mathrm{C}_{\mathrm{dl}}$ suggests that electrochemical surface area (ECSA) of the Geobacter/rGO electrode after the CA was significantly enhanced indicating higher degree of accessibility of catalytic sites.

Catalyst wettability is crucial in heterogeneous catalysis. ${ }^{49}$ Catalytic activity not only depends on the active sites, but also on the adsorption and desorption of the reactants and products. Hydrophilic surfaces can significantly improve catalysis by promoting a more catalytically favorable interaction between the electrode and electrolyte. A recent report proposed a strategy to enhance OER activity of NiFe hydroxide by simply improving surface hydrophilicity via phosphorylation. ${ }^{50}$ Zhou et al. observed a gradual increase in the wettability of fluorine and nitrogen co-doped carbon microsphere electrode with continuous $\mathrm{CV}$ measurements which improved the electrode capacitance. ${ }^{51}$ Such 
observations demonstrate that continuous electrochemical measurements can facilitate electrode wettability and supports the enhanced wettability of Geobacter/rGO electrode after the CA. All the above results (Fig. 5, Fig. S9 and S10) showed that after the CA, the interaction between the electrolyte and Geobacter/rGO electrode greatly improved due to the increased ECSA and surface hydrophilicity on the electrode. Furthermore, the CA treatment opened the OER catalytic active sites to the electrolyte, evidenced by the appearance of a pair of redox peaks in the CV of Geobacter/rGO (Fig. S10). On the other hand, TEM and Raman analyses of the Geobacter/rGO electrode showed no morphological changes after the CA measurements (Fig. S11 and S12) demonstrating that the structural character of the Geobacter/rGO catalyst was largely unchanged.

\section{Conclusions}

In summary, we have successfully demonstrated a unique single step approach for synthesizing doped/decorated rGO by G. sulfurreducens. Further, we demonstrated that the resulting Geobacter/rGO worked efficiently as an OER electrocatalyst with an overpotential of $270 \mathrm{mV}$ vs RHE producing a current density of $10 \mathrm{~mA} \mathrm{~cm}^{-2}$. This overpotential of $270 \mathrm{mV}$ is lower than those typically reported in studies using expensive benchmark metal oxide electrocatalysts such as $\mathrm{IrO}_{2}$ and $\mathrm{RuO}_{2}$. The approach used in this study is green and sustainable as it does not involve the use of toxic precursors and solvents. The OER activity observed in this report broadens the application of $G$. sulfurreducens as a cost-effective component of electrocatalyst manufacturing for large-scale energy conversion. Also, our work can inspire the use of other efficient EET-capable bacteria for synthesizing high-performing and low-cost electrocatalysts for various energy-related applications. 


\section{Acknowledgements}

This work was supported by Competitive Research Grant (URF/1/2985-01-01) from King Abdullah University of Science and Technology (KAUST).

\section{ASSOCIATED CONTENT}

\section{Supporting Information}

The Supporting Information is available free of charge on the ACS Publications website. The Supporting Information contains the details of ICP-OES data (Table S2 and Fig. S1), XPS analysis (Fig. S3-5 and Fig. S13), HRTEM images (Fig. S6 and Fig. S11), electrochemical data (Fig. S7-10) and Raman data (Fig. S12).

\section{AUTHOR INFORMATION}

\section{Corresponding Author}

*Phone: (+966) 544700129; e-mail: pascal.saikaly@kaust.edu.sa

\section{ORCID}

Pascal Saikaly: 0000-0001-7678-3986

\section{Authors Contributions}

S.K, K.P.K and P.E.S. conceived the idea and designed the project. All the authors wrote the paper. S.K synthesized Geobacter/rGO, conducted electrochemical tests, FTIR and contact angle measurements. K.P.K. cultured bacteria. A.A produced GO and $\mathrm{HrGO}$ and performed ICP-OES and Raman. S.P performed and analyzed XPS, SEM, EDS, designed graphical abstract, TOC and schematic illustrations in Fig. 1, Fig.3, and Fig. S2. N.K. captured TEM and SEM. P.E.S and P.C. supervised the work. All authors discussed the results and commented on the manuscript.

\section{Notes}

The authors declare no competing financial interests.

\section{References}

1.Roger, I.; Shipman, M. A.; Symes, M. D., Earth-abundant catalysts for electrochemical and photoelectrochemical water splitting. Nat. Rev. Chem. 2017, 1, (1).

2.Jung, S.; McCrory, C. C. L.; Ferrer, I. M.; Peters, J. C.; Jaramillo, T. F., Benchmarking nanoparticulate metal oxide electrocatalysts for the alkaline water oxidation reaction. J. Mater. Chem. A 2016, 4, (8), 3068-3076.

3.Zhao, Y.; Swierk, J. R.; Megiatto, J. D., Jr.; Sherman, B.; Youngblood, W. J.; Qin, D.; Lentz, D. M.; Moore, A. L.; Moore, T. A.; Gust, D.; Mallouk, T. E., Improving the efficiency of water splitting in dye-sensitized solar cells by using a biomimetic electron transfer mediator. Proc Natl Acad Sci US A 2012, 109, (39), 15612-6. 
4.Zheng, X.; Han, X.; Liu, H.; Chen, J.; Fu, D.; Wang, J.; Zhong, C.; Deng, Y.; Hu, W., Controllable Synthesis of Ni xSe $(0.5</=\mathrm{x}</=1)$ Nanocrystals for Efficient Rechargeable Zinc-Air Batteries and Water Splitting. ACS Appl Mater Interfaces 2018, 10, (16), 13675-13684.

5.Bian, B.; Alqahtani, M. F.; Katuri, K. P.; Liu, D.; Bajracharya, S.; Lai, Z.; Rabaey, K.; Saikaly, P. E., Porous nickel hollow fiber cathodes coated with CNTs for efficient microbial electrosynthesis of acetate from CO2 using Sporomusa ovata. J. Mater. Chem. A 2018, 6, (35), 17201-17211.

6.Katuri, K. P.; Kalathil, S.; Ragab, A. a.; Bian, B.; Alqahtani, M. F.; Pant, D.; Saikaly, P. E., DualFunction Electrocatalytic and Macroporous Hollow-Fiber Cathode for Converting Waste Streams to Valuable Resources Using Microbial Electrochemical Systems. Adv. Mater. 2018, 30, (26), 1707072. 7.Alqahtani, M. F.; Katuri, K. P.; Bajracharya, S.; Yu, Y.; Lai, Z.; Saikaly, P. E., Porous Hollow Fiber Nickel Electrodes for Effective Supply and Reduction of Carbon Dioxide to Methane through Microbial Electrosynthesis. Adv. Funct. Mater. 2018, 28, (43), 1804860.

8.Cui, X.; Ren, P.; Deng, D.; Deng, J.; Bao, X., Single layer graphene encapsulating non-precious metals as high-performance electrocatalysts for water oxidation. Energy Environ. Sci. 2016, 9, (1), 123-129.

9.Wang, H.; Maiyalagan, T.; Wang, X., Review on Recent Progress in Nitrogen-Doped Graphene: Synthesis, Characterization, and Its Potential Applications. ACS Catal. 2012, 2, (5), 781-794.

10.Lai, J.; Li, S.; Wu, F.; Saqib, M.; Luque, R.; Xu, G., Unprecedented metal-free 3D porous carbonaceous electrodes for full water splitting. Energy Environ. Sci. 2016, 9, (4), 1210-1214.

11.Qu, K.; Zheng, Y.; Dai, S.; Qiao, S. Z., Graphene oxide-polydopamine derived N, S-codoped carbon nanosheets as superior bifunctional electrocatalysts for oxygen reduction and evolution. Nano Energy 2016, 19, 373-381.

12.Paraknowitsch, J. P.; Thomas, A., Doping carbons beyond nitrogen: an overview of advanced heteroatom doped carbons with boron, sulphur and phosphorus for energy applications. Energy Environ. Sci. 2013, 6, (10).

13.Wang, J.; Ma, R.; Zhou, Z.; Liu, G.; Liu, Q., Magnesiothermic synthesis of sulfur-doped graphene as an efficient metal-free electrocatalyst for oxygen reduction. Sci Rep 2015, 5, 9304.

14.Groves, M. N.; Chan, A. S. W.; Malardier-Jugroot, C.; Jugroot, M., Improving platinum catalyst binding energy to graphene through nitrogen doping. Chem. Phys. Lett. 2009, 481, (4-6), 214-219.

15.Zhang, L.; Xia, Z., Mechanisms of Oxygen Reduction Reaction on Nitrogen-Doped Graphene for Fuel Cells. The Journal of Physical Chemistry C 2011, 115, (22), 11170-11176.

16.Zhao, J.; Liu, Y.; Quan, X.; Chen, S.; Zhao, H.; Yu, H., Nitrogen and sulfur co-doped graphene/carbon nanotube as metal-free electrocatalyst for oxygen evolution reaction: the enhanced performance by sulfur doping. Electrochim. Acta 2016, 204, 169-175.

17.Zhang, J.; Dai, L., Nitrogen, Phosphorus, and Fluorine Tri-doped Graphene as a Multifunctional Catalyst for Self-Powered Electrochemical Water Splitting. Angew. Chem. Int. Ed. Engl. 2016, 55, (42), 13296-13300.

18.Salas, E. C.; Sun, Z.; Lüttge, A.; Tour, J. M., Reduction of Graphene Oxide via Bacterial Respiration. ACS Nano 2010, 4, (8), 4852-4856.

19.Jiao, Y.; Qian, F.; Li, Y.; Wang, G.; Saltikov, C. W.; Gralnick, J. A., Deciphering the Electron Transport Pathway for Graphene Oxide Reduction by <span class="named-content genus-species" $\mathrm{id}=$ "named-content-1" $>$ Shewanella oneidensis $<$ /span $>$ MR-1. J. Bacteriol. 2011, 193, (14), 36623665 . 
20.Liu, T.; Jiang, L.-L.; He, M.-F.; Zhu, Z.; Wang, D.-b.; Song, T.-S.; Tan, W.-m.; Ouyang, P.; Xie, J., Green synthesis of reduced graphene oxide by a GRAS strain Bacillus subtilis 168 with high biocompatibility to zebrafish embryos. RSC Adv. 2015, 5, (74), 60024-60032.

21.Rathinam, N. K.; Berchmans, S.; Sani, R. K.; Salem, D. R., Rewiring the microbe-electrode interfaces with biologically reduced graphene oxide for improved bioelectrocatalysis. Bioresour. Technol. 2018, 256, 195-200.

22.Song, T.-S.; Tan, W.-M.; Xie, J., Bio-Reduction of Graphene Oxide Using Sulfate-Reducing Bacteria and Its Implication on Anti-Biocorrosion. Journal of Nanoscience and Nanotechnology 2018, 18, (8), 5770-5776.

23.Yoshida, N.; Miyata, Y.; Mugita, A.; Iida, K., Electricity Recovery from Municipal Sewage Wastewater Using a Hydrogel Complex Composed of Microbially Reduced Graphene Oxide and Sludge. Materials 2016, 9, (9), 742.

24.Yoshida, N.; Miyata, Y.; Doi, K.; Goto, Y.; Nagao, Y.; Tero, R.; Hiraishi, A., Graphene oxidedependent growth and self-aggregation into a hydrogel complex of exoelectrogenic bacteria. Sci. Rep. 2016, 6, 21867.

25.Wei, L.; Karahan, H. E.; Goh, K.; Jiang, W.; Yu, D.; Birer, Ö.; Jiang, R.; Chen, Y., A highperformance metal-free hydrogen-evolution reaction electrocatalyst from bacterium derived carbon. J. Mater. Chem. A 2015, 3, (14), 7210-7214.

26.Zhou, L.; Fu, P.; Wang, Y.; Sun, L.; Yuan, Y., Microbe-engaged synthesis of carbon dot-decorated reduced graphene oxide as high-performance oxygen reduction catalysts. J. Mater. Chem. A 2016, 4, (19), 7222-7229.

27.Malvankar, N. S.; Vargas, M.; Nevin, K. P.; Franks, A. E.; Leang, C.; Kim, B. C.; Inoue, K.; Mester, T.; Covalla, S. F.; Johnson, J. P.; Rotello, V. M.; Tuominen, M. T.; Lovley, D. R., Tunable metalliclike conductivity in microbial nanowire networks. Nat Nanotechnol 2011, 6, (9), 573-9.

28.Kalathil, S.; Pant, D., Nanotechnology to rescue bacterial bidirectional extracellular electron transfer in bioelectrochemical systems. RSC Adv. 2016, 6, (36), 30582-30597.

29.Malvankar, N. S.; Lovley, D. R., Microbial nanowires: a new paradigm for biological electron transfer and bioelectronics. ChemSusChem 2012, 5, (6), 1039-46.

30.Kumar, A.; Hsu, L. H.-H.; Kavanagh, P.; Barrière, F.; Lens, P. N. L.; Lapinsonnière, L.; Lienhard V, J. H.; Schröder, U.; Jiang, X.; Leech, D., The ins and outs of microorganism-electrode electron transfer reactions. Nat. Rev. Chem. 2017, 1, 0024.

31.Estevez-Canales, M.; Kuzume, A.; Borjas, Z.; Füeg, M.; Lovley, D.; Wandlowski, T.; EsteveNúñez, A., A severe reduction in the cytochrome $\mathrm{C}$ content of Geobacter sulfurreducens eliminates its capacity for extracellular electron transfer. Environ. Microbiol. Rep. 2015, 7, (2), 219-226.

32.Busalmen, J. P.; Esteve-Nunez, A.; Berna, A.; Feliu, J. M., C-type cytochromes wire electricityproducing bacteria to electrodes. Angew. Chem. Int. Ed. Engl. 2008, 47, (26), 4874-7.

33.Hunter, B. M.; Thompson, N. B.; Müller, A. M.; Rossman, G. R.; Hill, M. G.; Winkler, J. R.; Gray, H. B., Trapping an Iron(VI) Water-Splitting Intermediate in Nonaqueous Media. Joule 2018, 2, (4), 747-763.

34.Holmes, D. E.; Mester, T.; O'Neil, R. A.; Perpetua, L. A.; Larrahondo, M. J.; Glaven, R.; Sharma, M. L.; Ward, J. E.; Nevin, K. P.; Lovley, D. R., Genes for two multicopper proteins required for Fe(III) oxide reduction in Geobacter sulfurreducens have different expression patterns both in the subsurface and on energy-harvesting electrodes. Microbiology 2008, 154, (Pt 5), 1422-35. 
35.Marcano, D. C.; Kosynkin, D. V.; Berlin, J. M.; Sinitskii, A.; Sun, Z.; Slesarev, A.; Alemany, L. B.; Lu, W.; Tour, J. M., Improved Synthesis of Graphene Oxide. ACS Nano 2010, 4, (8), 4806-4814. 36.Alazmi, A.; Rasul, S.; Patole, S. P.; Costa, P. M. F. J., Comparative study of synthesis and reduction methods for graphene oxide. Polyhedron 2016, 116, 153-161.

37.Bond, D. R.; Lovley, D. R., Electricity Production by $<\mathrm{em}>$ Geobacter sulfurreducens $<$ /em $>$ Attached to Electrodes. Appl. Environ. Microbiol. 2003, 69, (3), 1548-1555.

38.Lin, W. C.; Coppi, M. V.; Lovley, D. R., $<$ em $>$ Geobacter sulfurreducens $</$ em $>$ Can Grow with Oxygen as a Terminal Electron Acceptor. Appl. Environ. Microbiol. 2004, 70, (4), 2525-2528.

39.Bansal, R.; Helmus, R. A.; Stanley, B. A.; Zhu, J.; Liermann, L. J.; Brantley, S. L.; Tien, M., Survival During Long-Term Starvation: Global Proteomics Analysis of Geobacter sulfurreducens under Prolonged Electron-Acceptor Limitation. J. Proteome Res. 2013, 12, (10), 4316-4326.

40.Estevez-Canales, M.; Kuzume, A.; Borjas, Z.; Fueg, M.; Lovley, D.; Wandlowski, T.; EsteveNunez, A., A severe reduction in the cytochrome $\mathrm{C}$ content of Geobacter sulfurreducens eliminates its capacity for extracellular electron transfer. Environ Microbiol Rep 2015, 7, (2), 219-26.

41.Krishnamoorthy, K.; Veerapandian, M.; Yun, K.; Kim, S. J., The chemical and structural analysis of graphene oxide with different degrees of oxidation. Carbon 2013, 53, 38-49.

42.Pimenta, M. A.; Dresselhaus, G.; Dresselhaus, M. S.; Cançado, L. G.; Jorio, A.; Saito, R., Studying disorder in graphite-based systems by Raman spectroscopy. PCCP 2007, 9, (11), 1276-1290.

43.Gangadharan, P. K.; Unni, S. M.; Kumar, N.; Ghosh, P.; Kurungot, S., Nitrogen-Doped Graphene with a Three-Dimensional Architecture Assisted by Carbon Nitride Tetrapods as an Efficient MetalFree Electrocatalyst for Hydrogen Evolution. CHEMELECTROCHEM 2017, 4, (10), 2643-2652.

44.Zhao, Y.; Nakamura, R.; Kamiya, K.; Nakanishi, S.; Hashimoto, K., Nitrogen-doped carbon nanomaterials as non-metal electrocatalysts for water oxidation. Nat Commun 2013, 4, 2390.

45.Yu, X.; Zhang, M.; Chen, J.; Li, Y.; Shi, G., Nitrogen and Sulfur Codoped Graphite Foam as a Self-Supported Metal-Free Electrocatalytic Electrode for Water Oxidation. Adv. Energy Mater. 2016, $6,(2)$.

46.Liu, M.; Zhang, R.; Chen, W., Graphene-supported nanoelectrocatalysts for fuel cells: synthesis, properties, and applications. Chem. Rev. 2014, 114, (10), 5117-60.

47.Suen, N.-T.; Hung, S.-F.; Quan, Q.; Zhang, N.; Xu, Y.-J.; Chen, H. M., Electrocatalysis for the oxygen evolution reaction: recent development and future perspectives. Chem. Soc. Rev. 2017, 46, (2), 337-365.

48.Zhou, H.; Yu, F.; Sun, J.; He, R.; Chen, S.; Chu, C.-W.; Ren, Z., Highly active catalyst derived from a $3 \mathrm{D}$ foam of $\mathrm{Fe}(\mathrm{PO}<\mathrm{sub}>3</$ sub $>)<$ sub $>2</$ sub $>/ \mathrm{Ni}<$ sub $>2</$ sub $>$ P for extremely efficient water oxidation. Proceedings of the National Academy of Sciences 2017, 114, (22), 5607-5611.

49.Wang, L.; Xiao, F.-S., The Importance of Catalyst Wettability. ChemCatChem 2014, 6, (11), 30483052.

50.Li, Y.; Zhao, C., Enhancing Water Oxidation Catalysis on a Synergistic Phosphorylated NiFe Hydroxide by Adjusting Catalyst Wettability. ACS Catal. 2017, 7, (4), 2535-2541.

51.Zhou, J.; Lian, J.; Hou, L.; Zhang, J.; Gou, H.; Xia, M.; Zhao, Y.; Strobel, T. A.; Tao, L.; Gao, F., Ultrahigh volumetric capacitance and cyclic stability of fluorine and nitrogen co-doped carbon microspheres. Nat Commun 2015, 6, 8503. 


\section{Table of contents}

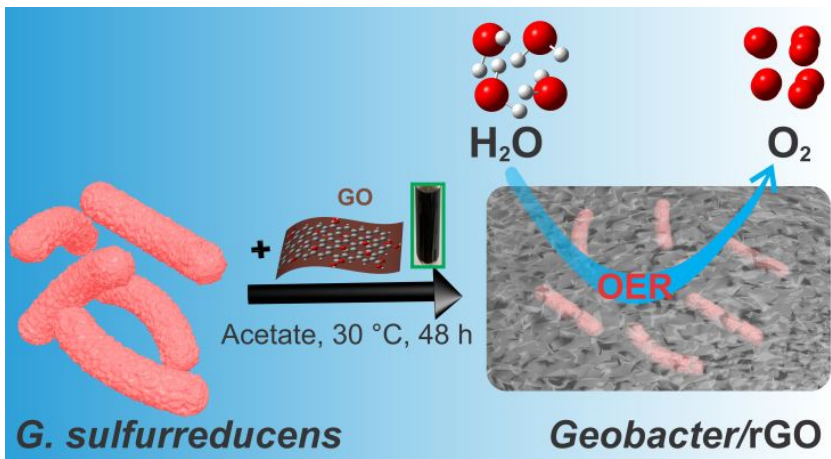

